\title{
Bichromatic Quadrangulations with Steiner Points
}

\author{
Victor Alvarez ${ }^{1}$, Toshinori Sakai ${ }^{2}$, Jorge Urrutia ${ }^{3}$ \\ 1 Facultad de Ciencias, Universidad Nacional Autónoma de México, México D.F., México. \\ 2 Research Institute of Educational Development, Tokai University, 2-28-4 Tomigaya, Shibuya- \\ ku, Tokyo 151-8677, Japan. \\ 3 Instituto de Matemáticas, Universidad Nacional Autónoma de México, México D.F., México.
}

\begin{abstract}
Let $P$ be a $k$ colored point set in general position, $k \geq 2$. A family of quadrilaterals with disjoint interiors $\mathcal{Q}_{1}, \ldots, \mathcal{Q}_{m}$ is called a quadrangulation of $P$ if $V\left(\mathcal{Q}_{1}\right) \cup \ldots \cup V\left(\mathcal{Q}_{m}\right)=P$, the edges of all $\mathcal{Q}_{i}$ join points with different colors, and $\mathcal{Q}_{1} \cup \ldots \cup \mathcal{Q}_{m}=\operatorname{Conv}(P)$. In general it is easy to see that not all $k$-colored point sets admit a quadrangulation; when they do, we call them quadrangulatable. For a point set to be quadrangulatable it must satisfy that its convex hull $\operatorname{Conv}(P)$ has an even number of points and that consecutive vertices of $\operatorname{Conv}(P)$ receive different colors. This will be assumed from now on.

In this paper we study the following type of questions: Let $P$ be a $k$-colored point set. How many Steiner points in the interior of $\operatorname{Conv}(P)$ do we need to add to $P$ to make it quadrangulatable? When $k=2$, we usually call $P$ a bichromatic point set, and its color classes are usually denoted by $R$ and $B$, i.e. the red and blue elements of $P$.

In this paper we prove that any bichromatic point set $P=R \cup B$ where $|R|=|B|=n$ can be made quadrangulatable by adding at most $\left\lfloor\frac{n-1}{3}\right\rfloor+\left\lfloor\frac{n}{2}\right\rfloor+1$ Steiner points and that $\frac{m}{3}$ Steiner points are occasionally necessary. To prove the latter, we also show that the convex hull of any monochromatic point set $P$ of $n$ elements can be always partitioned into a set $\mathcal{S}=\left\{\mathcal{S}_{1}, \ldots, \mathcal{S}_{t}\right\}$ of star-shaped polygons with disjoint interiors, where $V\left(\mathcal{S}_{1}\right) \cup \cdots \cup V\left(\mathcal{S}_{t}\right)=P$, and $t \leq\left\lfloor\frac{n-1}{3}\right\rfloor+1$. For $n=3 k$ this bound is tight. Finally we prove that there are 3 -colored point sets that cannot be completed to 3 -quadrangulatable point sets.
\end{abstract}

Key words. Triangulations, quadrangulations, bicolored point sets, Steiner points.

\section{Introduction}

Let $P=\left\{p_{1}, \ldots, p_{n}\right\}$ be a monochromatic point set in general position on the plane. A quadrangulation of $P$ is a set $\mathcal{Q}=\left\{\mathcal{Q}_{1}, \ldots, \mathcal{Q}_{m}\right\}$ of quadrilaterals (not necessarily convex) with disjoint interiors such that:

- $V\left(\mathcal{Q}_{1}\right) \cup \ldots \cup V\left(\mathcal{Q}_{m}\right)=P$, where $V(\mathcal{Q})$ denotes the set of vertices of a polygon $\mathcal{Q}$;

- $\mathcal{Q}_{1} \cup \ldots \cup \mathcal{Q}_{m}=\operatorname{Conv}(P)$ where $\operatorname{Conv}(P)$ denotes the convex hull of $P$ (see Figure 1 ).

The problem of finding quadrangulations of point sets has been studied for some time in the literature. It has been shown that quadrangulations are in some circumstances

\footnotetext{
* Supported by CONACYT of Mexico, Proyecto SEP-2004-Co1-45876, and PAPIIT (UNAM), Proyecto IN110802.
} 




Fig. 1. A quadrangulation of a point set. In this and all figures in this paper, red points are represented by small solid circles, and blue points by small open circles.

more desirable objects than triangulations, for example in the study of finite element methods and scattered data interpolation [7]. It is easy to see that not all point sets admit quadrangulations. It is known that a necessary and sufficient condition for a point set to admit a quadrangulation is that its convex hull has an even number of vertices [8, $2]$. If we add the condition that all elements of $\left\{\mathcal{Q}_{1}, \ldots, \mathcal{Q}_{m}\right\}$ are convex, the problems becomes more interesting. Such quadrangulations are called convex quadrangulations. It is easy to see that not all point sets (even with an even number of vertices in their convex hull) admit a convex quadrangulation. In [3] the problem of finding the minimum number of Steiner points needed to be added to a point set (located in the interior of $\operatorname{Conv}(P)$ ) so that the resulting collection admits a convex quadrangulation is studied. They proved that for some families of point sets $\left\lceil\frac{n-3}{2}\right\rceil-1$ Steiner points are necessary and $3\left\lfloor\frac{n}{2}\right\rfloor$ are always sufficient. The upper bound was recently improved to $4\left\lceil\frac{n}{5}\right\rceil+2$ Steiner points in [6]. For a more complete treatment of quadrangulations we recommend Toussaint's survey [10].

In this paper we study quadrangulations of colored point sets, that is point sets for which the elements of $P$ are partitioned into at least two non-empty disjoint subsets called the chromatic classes of $P$. More precisely, a set of quadrilaterals $\mathcal{Q}=\left\{\mathcal{Q}_{1}, \ldots, \mathcal{Q}_{m}\right\}$ with disjoint interiors is called a quadrangulation of $P$ if $V\left(\mathcal{Q}_{1}\right) \cup \ldots \cup V\left(\mathcal{Q}_{m}\right)=P$, $\mathcal{Q}_{1} \cup \ldots \cup \mathcal{Q}_{m}=\operatorname{Conv}(P)$ and the edges of all $\mathcal{Q}_{i}$ join elements of $P$ with different colors. It is clear that for a colored point set to be quadrangulatable, its convex hull must have an even number of vertices and consecutive vertices on the boundary of $\operatorname{Conv}(P)$ must receive different colors.

When the elements of $P$ are colored with two colors, it is customary to call the colors red and blue, and to call $P$ a bichromatic point set. The set of blue points of $P$ will be denoted by $B=\left\{b_{1}, \ldots, b_{s}\right\}$, and the set of red points by $R=\left\{r_{1}, \ldots, r_{t}\right\}$. We will assume that $P=R \cup B$ is in general position. It follows than that if $P$ is a bichromatic, quadrangulatable point set, its convex hull must have an even number of points such that their colors alternate between blue and red. It is also easy to see that the above condition is not sufficient [4]. In this paper we study the following question: How many Steiner points need to be added to any bichromatic point set such that the resulting point set is quadrangulatable?

In what follows we will assume that the convex hull of all bichromatic point sets $P=R \cup B$ considered here has an even number of points, and that their colors alternate 
between red and blue. We will also assume that all Steiner points to be added are invariably placed in the interior of $\operatorname{Conv}(P)$. Our main objective is to prove:

Theorem 1. Let $P=R \cup B$ be a bichromatic point set on the plane in general position such that $|R|=|B|=n>0$. Then we can always obtain a quadrangulatable bichro-

matic point set by adding most $\left\lfloor\frac{r}{2}\right\rfloor+1+\left\lfloor\frac{s-1}{3}\right\rfloor$ Steiner points to $P$. Moreover there are configurations for which $\frac{n}{3}$ Steiner points are always necessary.

To achieve the bounds in Theorem 1, some tight results on star-shaped partitionings of point sets were obtained that are interesting in their own right. A polygon $\mathcal{Q}$ is called star-shaped if there is a point $p \in \mathcal{Q}$ such that for any other point $q \in \mathcal{Q}$ the line segment connecting $p$ to $q$ is contained in $\mathcal{Q}$. A star-shaped partitioning of a point set $P$ is a set of star-shaped polygons $\mathcal{S}=\left\{\mathcal{S}_{1}, \ldots, \mathcal{S}_{t}\right\}$ with disjoint interiors such that $V\left(\mathcal{S}_{1}\right) \cup \ldots \cup V\left(\mathcal{S}_{t}\right)=P$, and $\mathcal{S}_{1} \cup \ldots \cup \mathcal{S}_{k}=\operatorname{Conv}(P)$. We prove that any point set $P$ always has a star-shaped partitioning with at most $\left\lfloor\frac{n-1}{3}\right\rfloor+1$ elements. This bound is tight for $n=3 k$.

To conclude this paper, a rather surprising result regarding quadrangulations of 3colored point sets will be proved. We show that there is a 3 -colored point set $P$ with 6 elements on its convex hull with consecutive elements of $P$ on the boundary of $\operatorname{Conv}(P)$ having different colors such that $P$ is not quadrangulatable, nor is it possible to add to $P$ any set $S$ of colored Steiner points points in the interior of $\operatorname{Conv}(P)$ such that $P \cup S$ is quadrangulatable (regarding the number, position and colors of the elements of $S$ ). Our result has a very similar flavor to Sperner's Lemma $[1,9]$.

\section{Lower bound}

In this section our objective is to prove the following result:

Lemma 1. There are bichromatic point sets with $6 k$ elements such that $2 k$ Steiner points must be added to them to make them quadrangulatable, $k \geq 2$.

Let us consider a convex polygon $\mathcal{C}$ with $2 k$ vertices labelled $v_{0}, \ldots, v_{2 k-1}$ in the clockwise direction. For each $i$, draw a line segment $\ell_{i}$ that intersects the edges $v_{i}-v_{i+1}$ and $v_{i+1}-v_{i+2}$, addition taken $\bmod 2 k$, in such a way that:

- $\ell_{i}$ intersects edge $v_{i}-v_{i+1}$ at a point close enough to $v_{i}$,

- $\ell_{i}$ intersects edge $v_{i+1}-v_{i+2}$ at a point close enough to $v_{i+1}$, and

$-\ell_{i}$ and $\ell_{i+1}$ intersect outside of $\mathcal{C}$.

For $i$ even (resp. $i$ odd), place two red points (resp. two blue points) on $\ell_{i}$ labelled $v_{i}^{\prime}$ and $v_{i}^{\prime \prime}$ close to each other, and close to the center of the segment $v_{i}-v_{i+1}, i+1 \bmod 2 k$. For $i$ even (resp. odd) color $v_{i}^{\prime}$ and $v_{i}^{\prime \prime}$ red (resp. blue); see Figure 2(a). Let $P$ be the set of $6 k$ points consisting of the vertices of $\mathcal{C}$ together with the blue and red points on the lines $\ell_{i}, i=0, \ldots, 2 k-1$.

Finally for $i=0, \ldots, 2 k-1$, let $\mathcal{P}_{i}$ be the polygons bounded by the edge $v_{i}-v_{i+1}$ and sectors of the edges $v_{i-1}-v_{i}, v_{i+1}-v_{i+2}$, a sector of the line segments joining $v_{i+1}$ to $v_{i}^{\prime}$, and another sector of the line passing through $v_{i}^{\prime}$ and $v_{i}^{\prime \prime}$ as shown in Figure 2(b).

We now proceed to prove the lower bound of Theorem 1. 


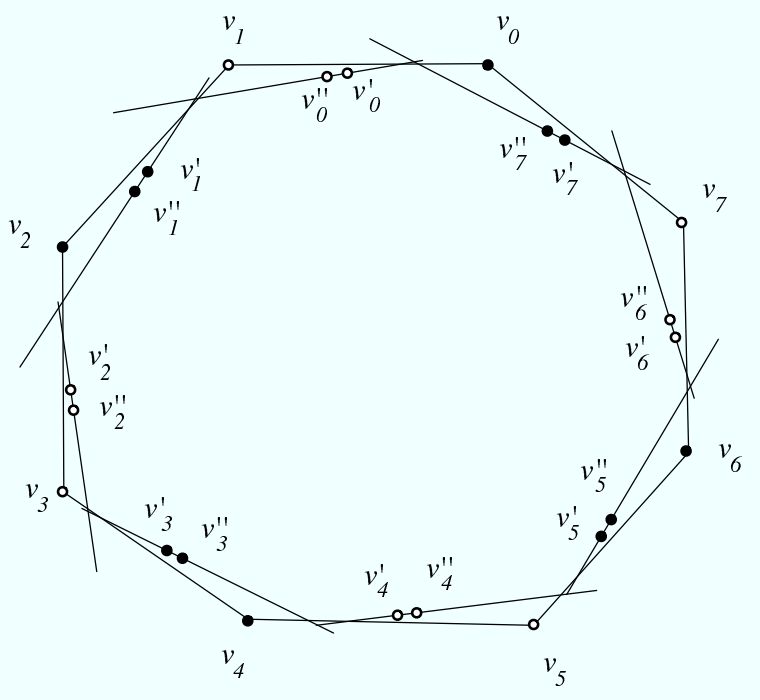

(a)



(b)

Fig. 2.

Proof. Let us note that in any quadrangulation $\mathcal{Q}$ of $P$, each edge $v_{i}-v_{i+1}$ of $\mathcal{C}$ is an edge of exactly one quadrilateral $\mathcal{Q}_{i} \in \mathcal{Q}$. It is easy to see that at least one vertex of each $\mathcal{Q}_{i}$ is a Steiner point that belongs to the interior of $\mathcal{P}_{i}$ (see Figure 3). We now show that for each $\mathcal{Q}_{i}$ we can find a Steiner point $p_{i} \in \mathcal{P}_{i}$ such that for $i \neq j$, we have that $p_{i} \neq p_{j}$.
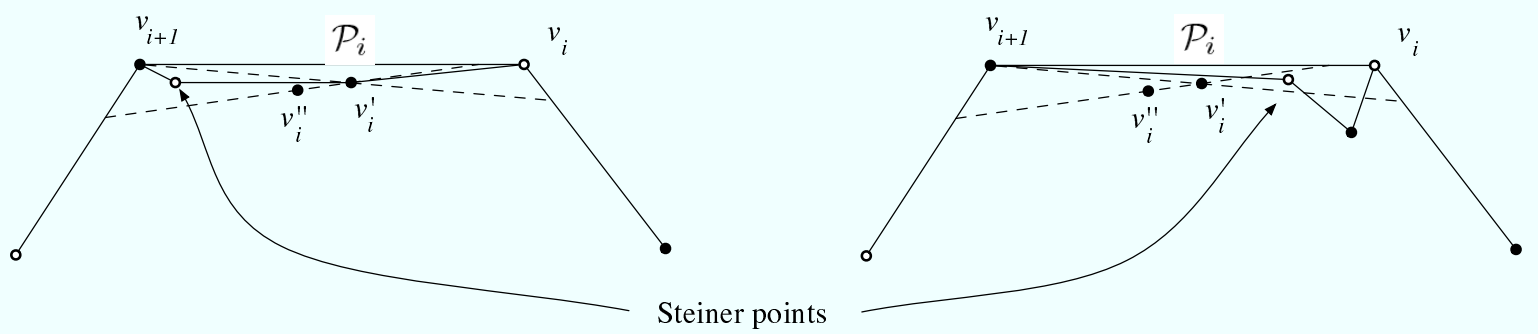

Fig. 3.

Observe first that for $i \neq j \pm 1, \mathcal{P}_{i} \cap \mathcal{P}_{j}=\emptyset$, therefore any Steiner point $p_{i}$ we may choose for $\mathcal{Q}_{i}$ is different from any Steiner point $p_{j}$ chosen for $\mathcal{Q}_{j}$. Therefore we focus only on Steiner points in polygons $\mathcal{Q}_{i}$ and $\mathcal{Q}_{i+1}$.

Consider two consecutive edges $v_{i}-v_{i+1}$ and $v_{i+1}-v_{i+2}$ of $\mathcal{C}$, and assume that we are forced to choose $p_{i}=p_{i+1}=p$. Observe that $p \in \mathcal{P}_{i} \cap \mathcal{P}_{i+1}$, and thus it is close to $v_{i+1}$. Assume w.l.o.g. that $v_{i}$ is red and therefore $v_{i+1}$ is blue. Two cases arise:

1. The color of $p_{i}$ is blue; see Figure 4(a).

In this case, it must happen that $p_{i}-v_{i}$ and $p_{i}-v_{i+2}$ are edges of $\mathcal{Q}_{i}$ and $\mathcal{Q}_{i+1}$. If the quadrilateral $\mathcal{X}$ with vertices $\left\{v_{i}, v_{i+1}, v_{i+2}, p_{i}\right\}$ does not contain any element of the set of $\left\{v_{i}^{\prime}, v_{i}^{\prime \prime}, v_{i+1}^{\prime}, v_{i+1}^{\prime \prime}\right\}$, we obtain a configuration similar to the original one, and we are introducing redundant Steiner points; see Figure 4. Therefore it must happen that 


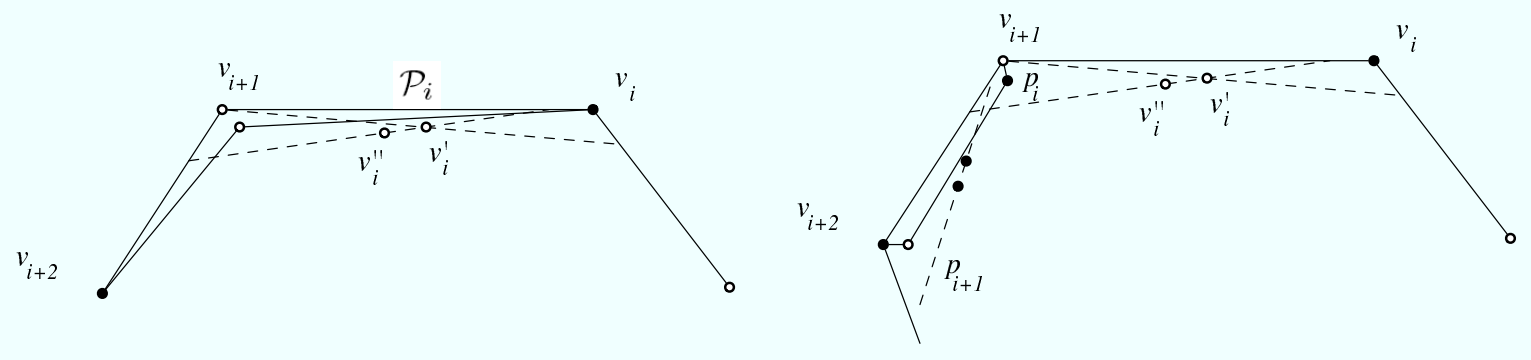

(a)

(b)

Fig. 4.

at least one of $\left\{v_{i}^{\prime}, v_{i}^{\prime \prime}, v_{i+1}^{\prime}, v_{i+1}^{\prime \prime}\right\}$ belongs to the interior of $\mathcal{X}$, and we need to have a second Steiner point $q$ in the interior of $\mathcal{X}$. It is easy to see that $p_{i}$ and $q$ cannot be associated to any other $\mathcal{Q}_{j}, j \neq i, i+1$. Change $p_{i}$ to $q$, let $p_{i+1}$ be $p_{i}$, and we are done.

2. The color of $p_{i}$ is red; see Figure $4(\mathrm{~b})$.

This is a harder case to solve. Observe first that $p_{i}$ is necessarily adjacent to $v_{i+1}$. Moreover the fourth vertex of $\mathcal{Q}_{i}$ must be a blue Steiner point $b$ contained in $P_{i+1}$. If $b \notin \mathcal{P}_{i+1} \cap \mathcal{P}_{i+2}$ then we can let $p_{i+1}=b$ and we are done.

If $p \in \mathcal{Q}_{i+1} \cap \mathcal{Q}_{i+2}$, then we can apply the same argument as before, but to $v_{i+1}$ and $r=p_{i+1}$ instead of $v_{i}$ and $p_{i}$ (i.e. the fourth vertex of $\mathcal{Q}_{i+2}$ is a Steiner point on the interior of $P_{i+1}$ etc.) Repeat this until an index $j$ is reached such that $p_{j} \notin \mathcal{P}_{j} \cap \mathcal{P}_{j+1}$ or we get back to $\mathcal{Q}_{i}$. In the first case, we can conclude in a similar way to when $j=i+1$, we can choose $p_{i}, \ldots, p_{j}$ such that they are all different, and that for $k \notin\{i, \ldots, j\}$ $p_{k} \neq p_{\ell}, \ell \in\{i, \ldots, j\}$. In the second case, we obtain a different $p_{i}$ for each $i$.

Therefore we have shown that a different Steiner point can be assigned to each $\mathcal{Q}_{i}$, and thus the number of Steiner points need to be added to $P$ is at least $2 k$.

\section{Upper bound}

Before we can proceed with the proof for the upper bound of Theorem 1, we need the following result, which is interesting in its own right:

Theorem 2. Let $P$ be a point set in general position on the plane, where $|P|=n$. Then $P$ has a star-shaped partitioning $\mathcal{S}=\left\{\mathcal{S}_{1}, \ldots, \mathcal{S}_{t}\right\}$ with at most $t \leq\left\lfloor\frac{n-1}{3}\right\rfloor+1$ elements. For $n=3 k$, this bound is tight.

Proof. Let $q_{0}$ be the leftmost vertex of $\operatorname{Conv}(P)$ and let $x, y$ be the counterclockwise and clockwise neighbors of $q_{0}$ in $\operatorname{Conv}(P)$ respectively.

Let us relabel the points in the interior of $\operatorname{Conv}(P)$ together with $x, y$ by $q_{1}=$ $x, q_{2}, \ldots, q_{r-1}, q_{r}=y$ such that for $i<j$, the slope of the straight line segment joining $q_{0}$ to $q_{i}$ is smaller than that of the segment joining $q_{0}$ to $q_{j}$. Finally let $q_{r+1}, q_{r+2}, \ldots, q_{n-1}$ be the remaining points of $P-\left\{q_{0}, q_{1}, q_{r}\right\}$ on the boundary of $\operatorname{Conv}(P)$ labeled in clockwise order such that $q_{r+1}$ is the neighbor of $q_{r}$ on $\operatorname{Conv}(P)$; see Figure 5.

Let $\mathcal{S}_{1}$ be the star-shaped polygon with vertex set $\left\{q_{0}, q_{1}, \ldots, q_{r-1}, q_{r}\right\}$ and edge set $\left\{q_{i} q_{i+1}\right\}, 0 \leq i \leq r$ with addition modulo $r+1$. 




Fig. 5.

Let $\mathcal{P}^{\prime}$ be the polygon with vertex set $\left\{q_{1}, \ldots, q_{r}, q_{r+1}, \ldots, q_{n-1}\right\}$ and edge set $\left\{q_{i} q_{i+1}\right\} \cup$ $\left\{q_{n-1} q_{1}\right\}$ where $1 \leq i \leq n-2$. See Figure 5 .

Following Fisk's [5] proof of Chvátal's classical Art Gallery Theorem, let $T$ be a triangulation of $\mathcal{P}^{\prime}$. We can now 3 -color the vertices of $\mathcal{P}^{\prime}$ such that every two vertices $q_{i}$ and $q_{j}$ that are joined by an edge of $T$ receive different colors; see Figure 6 . Since $\left|V\left(\mathcal{P}^{\prime}\right)\right|=n-1$, the smallest chromatic class has at most $\left\lfloor\frac{n-1}{3}\right\rfloor$ vertices. Let $v_{1}, \ldots, v_{s}, 1 \leq s \leq\left\lfloor\frac{n-1}{3}\right\rfloor$, be the vertices of such a chromatic class.
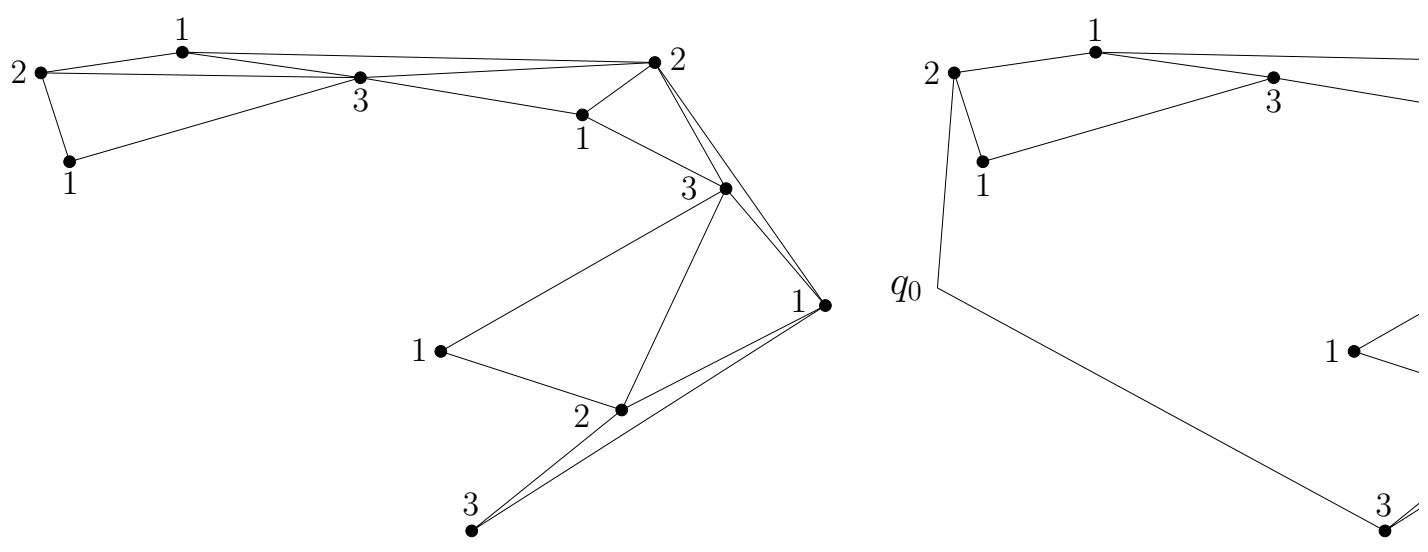

Fig. 6.

For each $v_{i}, 1 \leq i \leq s$, construct the polygon $\mathcal{S}_{i+1}$ which is the union of all triangles in which $v_{i}$ is a vertex. Clearly each $\mathcal{S}_{i+1}$ is a star-shaped polygon, since all of its boundary is visible from $v_{i}$. Clearly the set $\left\{\mathcal{S}_{i+1}: 1 \leq s \leq\left\lfloor\frac{n-1}{3}\right\rfloor\right\}$ together with $\mathcal{S}_{1}$ form a star-shaped partitioning of $P$ with at most $\left\lfloor\frac{n-1}{3}\right\rfloor+1$ polygons, and our result follows. See Figure 6 .

We now show that this bound is tight.

Let $\mathcal{C}$ be a convex polygon with $2 k$ edges labeled clockwise $e_{1}, e_{2}, \ldots, e_{2 k}$. For each edge $e_{i}$ with $i \in O d d=\{1,3,5, \ldots, 2 k-1\}$, place a point labeled $q_{i}$ in the interior of $\mathcal{C}$ and very close to the midpoint of $e_{i}$. The vertices of $\mathcal{C}$ together with the points $q_{i}, i \in \operatorname{Odd}$, generate a point set $Q$ with $3 k$ elements. For each $i \in O d d$, consider the triangle whose vertices are the endpoints of $e_{i}$ together with $q_{i}$; see Figure 7.

It is easy to see that in any star-shaped partition of $\operatorname{Conv}(Q)$, every polygon contains at most one of these triangles (and must contain all of it). Our result follows. 


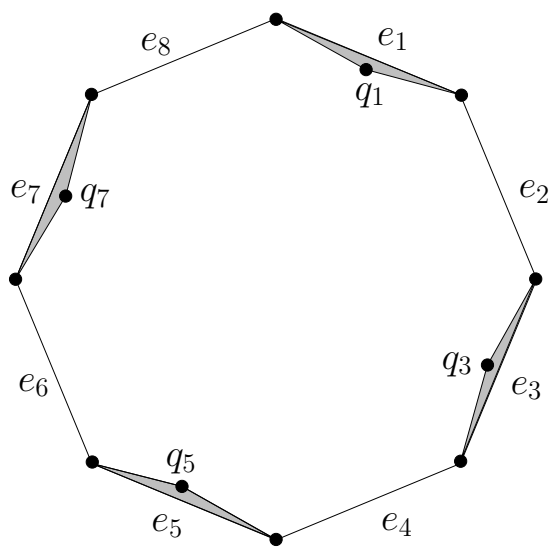

Fig. 7. The shaded triangles must necessarily be in different star-shaped polygons.

3.0.1. Proof of Theorem 1 Another result must be proved before tackling the proof of Theorem 1. Recall that a vertex $v$ of a polygon $\mathcal{Q}$ is called reflex if the internal angle of $\mathcal{Q}$ at $v$ is greater than 180 degrees.

Let $\mathcal{Q}$ be a bichromatic non-convex quadrilateral. Clearly $\mathcal{Q}$ contains exactly one reflex vertex. Suppose that the reflex vertex of $\mathcal{P}$ is red, and that there are $k$ blue points in the interior of $\mathcal{P}$. We address the following question: How many red Steiner points are needed such that $\mathcal{Q}$ can be quadrangulated?

Observe that if there is exactly one blue point in the interior of $\mathcal{Q}$, no Steiner points need to be added. However if there are exactly two blue points in the interior of $\mathcal{Q}$, one Steiner point might be needed; see Figure 8. In general it is easy to see that if there are $k$ blue points in the interior of $\mathcal{Q}$, it can always be quadragulated using at most $\left\lfloor\frac{k}{2}\right\rfloor$ red Steiner points. Thus we do not give a proof of the following result:

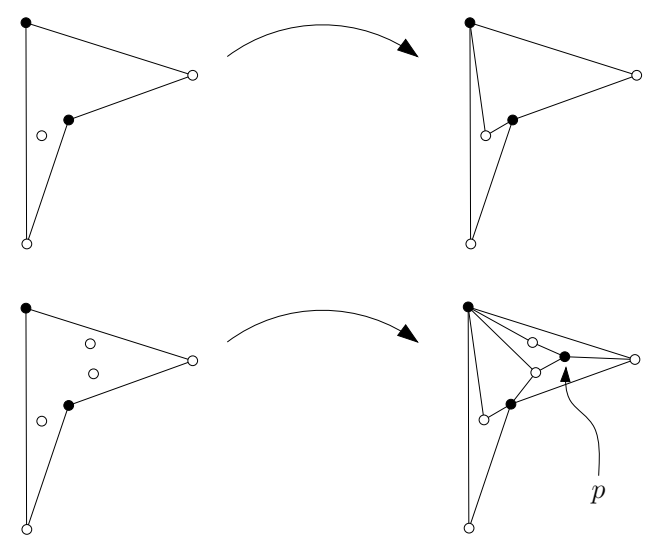

Fig. 8.

Lemma 2. Let $\mathcal{Q}$ be a non-convex bichromatic quadrilateral such that the reflex vertex of $\mathcal{Q}$ is red (resp. blue), and it contains $k$ blue (resp. red) points in its interior. Then $\mathcal{Q}$ can be quadrangulated using at most $\left\lfloor\frac{k}{2}\right\rfloor$ Steiner points placed in its interior.

We proceed now to prove the upper bound of Theorem 1.

Proof. Let us recall that we are assuming that the elements of $P$ on the boundary of $\operatorname{Conv}(P)$ alternate in color. Temporarily remove all the points of $B$ including those on 
the boundary of $\operatorname{Conv}(P)$ (they will be replaced later). Using Theorem 2, $\operatorname{Conv}(R)$ can be partitioned into a set $\mathcal{S}=\left\{\mathcal{S}_{1}, \ldots, \mathcal{S}_{t}\right\}, 1 \leq t \leq\left|\frac{n-1}{3}\right|+1$, of star-shaped polygons. See Figure 9.

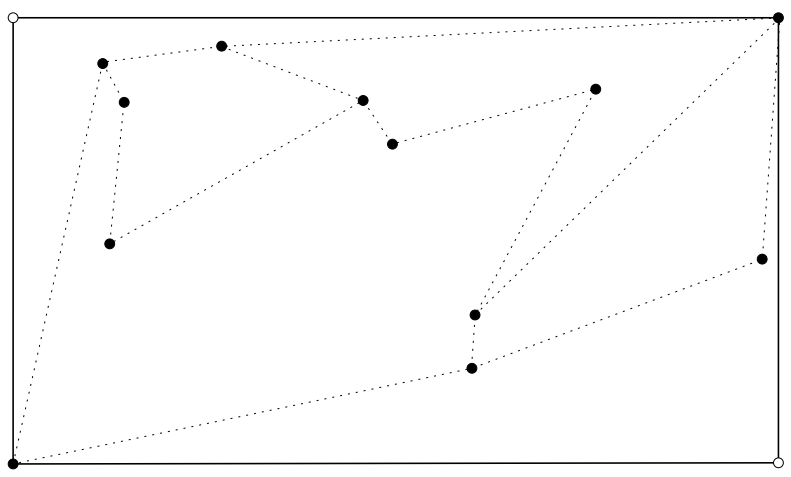

Fig. 9.

In the interior of each $\mathcal{S}_{i}$ add a Steiner point $p_{i}$ from which all of the boundary of $\mathcal{S}_{i}$ is visible. Color $p_{i}$ blue and join it to each vertex of $\mathcal{S}_{i}$ by a straight line segment.

Observe that for each $\mathcal{S}_{i}$, all of its edges, except those on the boundary of $\operatorname{Conv}(R)$, are diagonals of bichromatic quadrilaterals; see Figure 10. Replace the set $B^{\prime}$ of blue points of $P$ that lie on the boundary of $\operatorname{Conv}(P)$. Observe that each edge $e_{i}$ of the polygon defined by the boundary of $\operatorname{Conv}(R)$ is visible from exactly one element $p \in B^{\prime}$, and that by joining the ends of $e_{i}$ to $p$, we construct a bichromatic quadrangulation of $B^{\prime} \cup R \cup\left\{p_{i}, 0 \leq i \leq t\right\}$ in which the edges of all $\mathcal{S}_{i}, 0 \leq i \leq t$ are diagonals of the quadrilaterals; see Figure 11. Removing all the edges of all $\mathcal{S}_{i}, 0 \leq i \leq s$, results in a bichromatic quadrangulation $\mathcal{K}$ that uses all the points in $R$ plus at most $\left\lfloor\frac{n-1}{3}\right\rfloor+1$ blue Steiner points; see Figure 12.

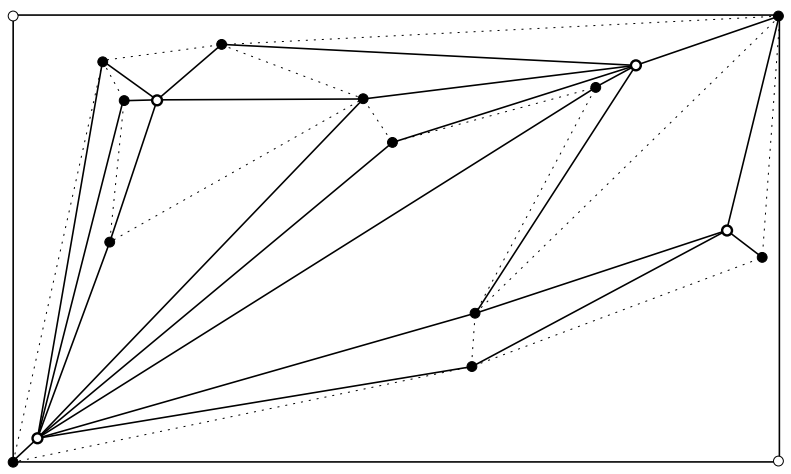

Fig. 10.

Now return all the remaining elements of $B-B^{\prime}$ to their original position and proceed to finish the quadrangulation. Let $\mathcal{F}_{i}$ be any face of $\mathcal{K}$. By Lemma 2 , if $\mathcal{F}_{i}$ contains $b_{i}$ elements of $B-B^{\prime}, \mathcal{F}_{i}$ can be quadrangulated by adding at most $\left\lfloor\frac{b_{i}}{2}\right\rfloor$ Steiner points. Observe that

$$
\sum_{F_{i} \in \mathcal{K}} b_{i} \leq n-2 .
$$




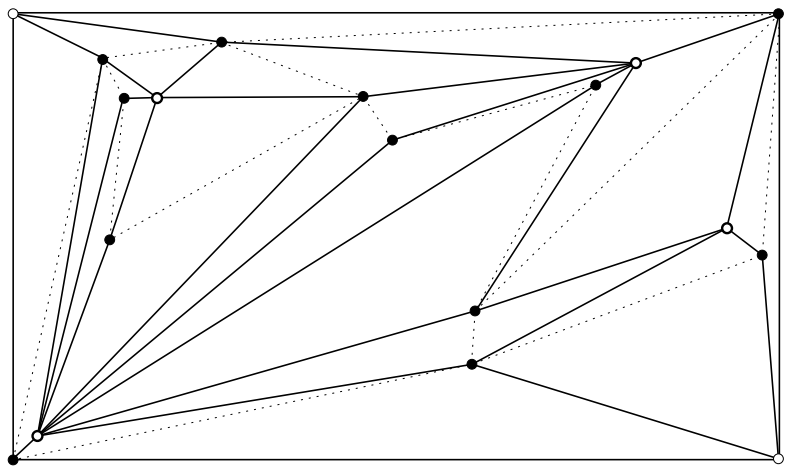

Fig. 11.

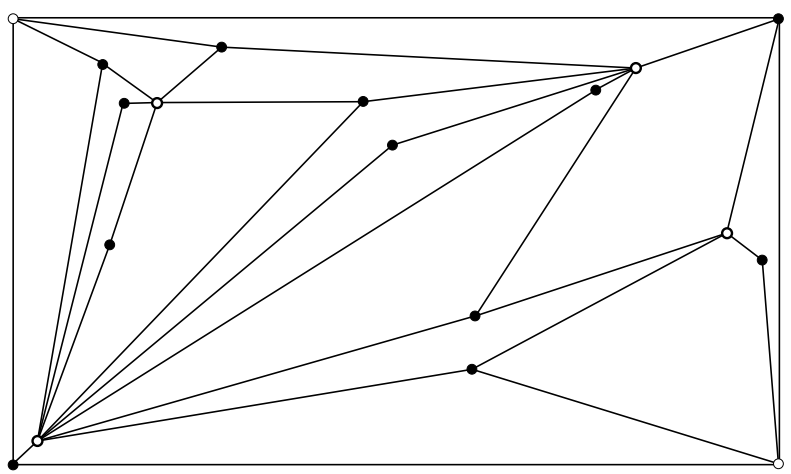

Fig. 12.

And thus

$$
\sum_{F_{i} \in \mathcal{K}}\left\lfloor\frac{b_{i}}{2}\right\rfloor \leq\left\lfloor\frac{n-2}{2}\right\rfloor
$$

The worst case arises when all $b_{i}$ are 0 or 2 . Thus the number of Steiner points used is at most $\left\lfloor\frac{n-1}{3}\right\rfloor+\left\lfloor\frac{n-2}{2}\right\rfloor+1 \approx 5 \frac{m}{12}$ and our result follows. Figure 14 shows the final quadrangulation.



Fig. 13.

\section{Beyond 2-colorings}

In this section we will study quadrangulations of 3-colored point sets. For the sake of clarity, the colors used will now be labeled by the set of integers $I_{3}=\{1,2,3\}$. 


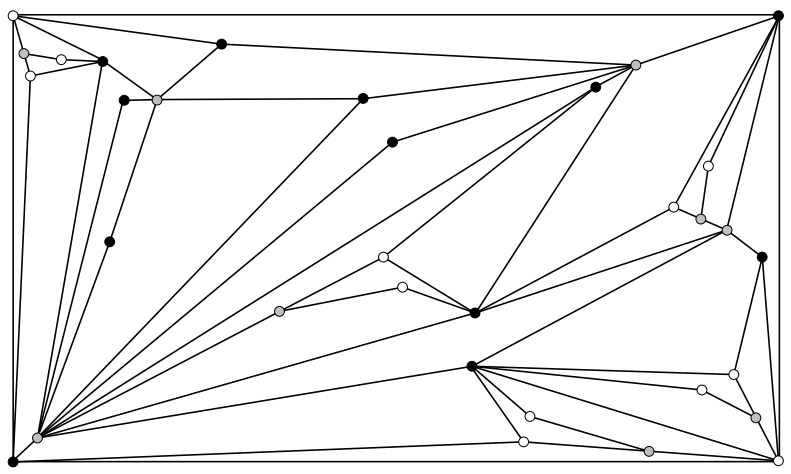

Fig. 14. The final quadrangulation using $\approx 5 \frac{m}{12}$ Steiner points, which are shown in gray.

Let $P$ be a set of $n$ points on the plane in general position. Let us assume that each element of $P$ is assigned a single color in the set $I_{3}$. Recall that if a 3-colored point set is quadrangulatable, its convex hull must have an even number of vertices, and adjacent vertices on $\operatorname{Conv}(P)$ must have different colors. In the rest of this section, we will assume that our point sets satisfy these conditions.

Not all 3-colored point sets are 3-quadrangulatable. Let $P_{6}$ be the 3-colored point set $\left\{p_{0}, \ldots, p_{5}\right\}$ consisting of the set of vertices of a convex hexagon $H$ (labeled in clockwise order along the boundary of $H$ ) such that $p_{0}$ and $p_{3}$ are colored with color $1, p_{1}$ and $p_{4}$ with color 2 , and $p_{3}$ and $p_{5}$ with color 3 ; see Figure $15(\mathrm{a})$. It is easily to verify that $P_{6}$ is not 3-quadrangulatable.

The final quadrangulation using $\approx 5 \frac{m}{12}$ Steiner points, which are shown in gray.

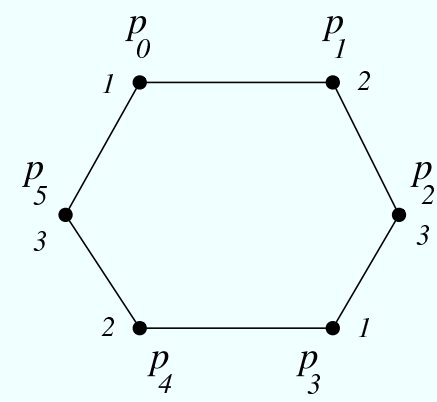

(a)

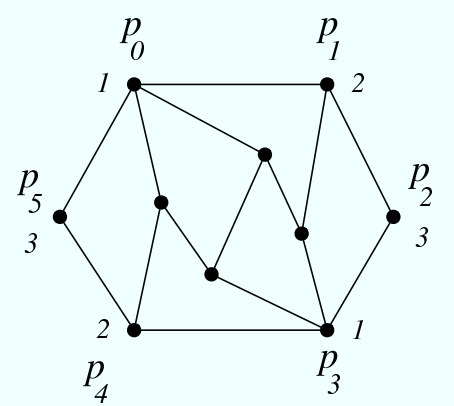

(b)

Fig. 15.

In this section we study the following problem:

Problem 1. If a 3-colored point set $P$ is not quadrangulatable, how many colored Steiner points must be added to $P$ to obtain a 3 -colored quadrangulatable point set?

To our surprise, the answer to this question is:

Theorem 3. There is a 3-colored point set $P$ (namely $P_{6}$ ) such that it is not possible to add any 3-colored point set $S$ to $P$ such that all the elements of $S$ belong to the interior of $\operatorname{Conv}\left(P_{6}\right)$, and such that $P \cup S$ is quadrangulatable (regardless of the number, position and colors of the elements of $S$ ). 
Let $S$ be a set of points contained in the interior of $\operatorname{Conv}\left(P_{6}\right)$ (for now $S$ is not a colored point set). Consider for a moment $P_{6} \cup S$ as a monochromatic point set, and let $\mathcal{Q}$ be a quadrangulation of $P=P_{6} \cup S$; see Figure 15(b). That such a quadrangulation always exists was proved in [10]. Let $G$ be the graph whose vertex is $P_{6} \cup S$ in which two vertices are adjacent iff they are joined by an edge of $\mathcal{Q}$; see Figure 15(b).

We now prove:

Theorem 4. In any coloring of $G$ such that $p_{0}$ and $p_{3}$ are colored $1, p_{1}$ and $p_{4}$ are colored 2 , and $p_{2}$ and $p_{5}$ are colored 3 , there is always an edge $e$ of $G$ such that the two vertices of e receive the same color.

Proof. Let us consider any coloring of the vertices of $G$ with colors $\{1,2,3\}$. Observe that here we do not require a good coloring of $G$, i.e. a coloring of the vertices of $G$ such that adjacent vertices receive different colors. Let $H$ be the dual graph of $G$, that is for each face of $G$ let us put a vertex in $H$, and for each edge $e$ of $G$ let us put an edge in $H$ connecting the vertices representing the faces of $G$ that contain $e$ on their boundaries. Let $v_{\infty}$ denote the vertex in $H$ representing the unbounded face of $G$. See Figure 16(a). In general $H$ may have multiple edges; for example the dual of the quadrangulation in Figure 1 has several such edges.



(a)

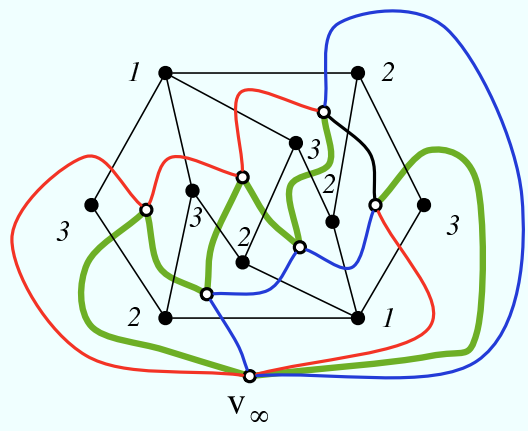

(b)
Color 1

Color 2

Color 3

Color 0



Fig. 16.

Let us define a sum $\oplus$ in $I_{3}$ as follows:

$$
i \oplus i=0, \quad 1 \oplus 2=3, \quad 1 \oplus 3=2, \quad \text { and } 2 \oplus 3=1 .
$$

We now color the edges of $H$ as follows: an edge $e$ of $H$ crossing an edge $f$ of $G$ whose vertices are colored $i$ and $j$ will be colored with color $i \oplus j$. See Figure 16(b).

We now prove that there must be an edge of $H$ whose color is 0 , that is, there is an edge in $G$ such that both of its vertices have the same color.

Suppose all edges of $G$ have their vertices colored with different colors. Consider a quadrilateral $\mathcal{Q}$ of $G$. It is easy to see that either

1. the four edges of $H$ crossing the edges of $\mathcal{Q}$ have all the same color, or

2. the four edges of $H$ crossing the four edges of $\mathcal{Q}$ have exactly two colors, say $i$ and $j$, and two of these edges have color $i$ and two color $j$. Moreover the curves obtained by joining edges of the same color do not cross each other, they only meet at the vertex representing $\mathcal{Q}$ in $H$. 


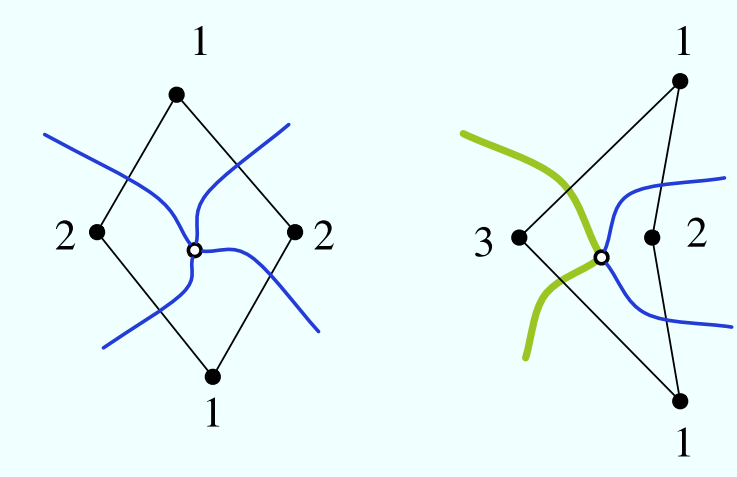

Fig. 17.

The first case arises when the vertices of $\mathcal{Q}$ receive only two colors, and the second when they receive three different colors; see Figure 17. We are ready to finish the proof. Let $H_{i}$ be the subgraph of $H$ containing all the edges of $H$ of color $i, i=1, \ldots, 3$. Note that because of the above observations, all vertices of each $H_{i}$ have even degree, that is degree 2 or 4 . Also for each $i, v_{\infty}$ is a vertex of $H_{i}$. Moreover, let $C_{i}$ and $C_{j}$ be simple closed curves obtained by joining edges of $H_{i}$ and $H_{j}$ respectively. By the second observation above, $C_{i}$ and $C_{j}$ do not cross in the interior of $\operatorname{Conv}\left(P_{6}\right)$. Then since all the vertices of $H_{1}$ and $H_{2}$ are even, there is a cycle in each of them containing $v_{\infty}$; let us call these $C_{1}$ and $C_{2}$ respectively. Since $C_{1}$ and $C_{2}$ cross at $v_{\infty}$, by the Jordan Curve Theorem they must cross again. However the second crossing of $C_{1}$ and $C_{2}$ must be in the interior of $\operatorname{Conv}\left(P_{6}\right)$ which contradicts the second observation. The result follows.

We can now proceed with the proof of Theorem 3 .

Proof. Suppose that there is a 3-chromatic set $S$ contained in the interior of $\operatorname{Conv}\left(P_{6}\right)$ such that $P_{6} \cup S$ is quadrangulatable. Let $\mathcal{Q}$ be any quadrangulation of $P_{6} \cup S$. Then the graph $G$ with vertex set $P_{6} \cup S$ generated by $\mathcal{Q}$ is 3 -chromatic, contradicting Theorem 4 .

\section{Conclusions}

In this paper we studied bichromatic and 3-chromatic quadrangulations of colored point sets on the plane. We proved that if a bicolored point set has an even number of vertices on its convex hull, and consecutive vertices on $\operatorname{Conv}(P)$ have different colors, then by adding at most $\left\lfloor\frac{n-1}{3}\right\rfloor+\left\lfloor\frac{n-2}{2}\right\rfloor+1 \approx 5 \frac{m}{12}$ Steiner points in the interior of $\operatorname{Conv}(P)$ we can make $P$ quadrangulatable, with $\left\lceil\frac{n}{3}\right\rceil$ points sometimes necessary. An interesting problem is that of closing the gap between the upper and lower bounds. We believe that the tight bound is the lower bound.

While trying to generalize our results for more than two colors, we proved a surprising result similar in flavor to Sperner's Lemma [1,9]. Let $H$ be a convex hexagon, $S$ a point set contained in the interior of $H$, and let $Q$ be any quadrangulation of $H \cup S$. Then in any 3-coloring of of the vertices of $Q$ such that the vertices of $H$ are colored in the clockwise order $1,2,3,1,2,3$, there is an edge of $Q$ whose vertices have the same color. 


\section{References}

1. A. Bondy, and U.S.R. Murty, Graph Theory with Applications, Elsevier Science Publishing Co. New York, 1976.

2. P. Bose and G. Toussaint. Characterizing and efficiently computing quadrangulations of planar point sets. Computer Aided Geometric Design, 14, (1997) 763-785.

3. D. Bremner, F. Hurtado, S. Ramaswami and V. Sacristán. Small convex quadrangulations of point sets. Algorithmica, 38:2, (2003) 317-339.

4. C. Cortés, A. Márquez, A. Nakamoto and J. Valenzula. Quadrangulations and 2-colorations. 21st European Workshop on Computational Geometry, Eindhoven, March 9-11, (2005) 65-68.

5. S. Fisk, A short proof of Chvátal's watchman theorem. Journal of Combinatorial Theory, Series B, 24, (1978) 374.

6. M. Heredia and J. Urrutia. On convex quadrangulations of point sets on the plane. To appear in LNCS, Proc. China-Japan Joint Conference on Discrete Geometry, Combinatorics and Graph Theory 2005.

7. M.L. Lai and L.L. Schumaker. Scattered data interpolation using piecewise polynomials of degree six. SIAM Journal on Numerical Analysis, 34, (1997) 905-921.

8. S. Ramaswami, P. Ramos and G. Toussaint. Converting triangulations to quadrangulations. Computational Geometry: Theory And Applications, 9, (1998) 257-276.

9. E. Sperner. Neuer Beweis für die Invarianz der Dimensionszahl und des Gebietes. Hamburger Abhand. 6, 265-272 (1928).

10. G. Toussaint. Quadrangulations of planar sets. In Proceedings of the 4th International Workshop on Algorithms and Data Structures, 218-227. Springer-Verlag, 1995. 